\title{
Convergence to local random attractors
}

\author{
Peter Ashwin \\ School of Mathematical Sciences, \\ University of Exeter, \\ Exeter EX4 4QE, \\ United Kingdom \\ P.Ashwin@ex.ac.uk
}

\author{
Gunter Ochs \\ Institut für Dynamische Systeme, \\ Fachbereich 3, \\ Universität Bremen, \\ Postfach 330 440, \\ D28334 Bremen, Germany \\ gunter@math . uni-bremen.de
}

January 29, 2003

\begin{abstract}
Random attractors allow one to classify qualitative and quantitative aspects of the long-time behaviour of stochastically-forced systems viewed as random dynamical systems (RDS) in an analogous way to attractors for deterministic systems.

We compare several notions of random attractor in RDS by examining convergence of trajectories to a random invariant set in different ways, including convergence of mean distance, convergence in probability and convergence on dense subsequences as well as pullback convergence. We give examples showing how these concepts of attraction are inequivalent.

We also examine definitions for local attractors and possible methods of decomposition of random attractors into 'random Milnor' attractors. We point out some problems that remain in interpreting these.

Finally, we examine numerical simulations of a stochastic van der Pol-Duffing equation and find cases where there appear to be Milnor attractors with positive top Lyapunov exponents. We explain bursting behaviour of the two-point motion for some parameter values in terms of the presence of nontrivial random Milnor attractors.
\end{abstract}

\section{Introduction}

The long time asymptotic dynamics of deterministic dynamical systems typically takes place on a small subset of the phase space referred to as an attractor for the system. There are various notions of attraction, for example asymptotically stable attractors attract open sets of initial conditions whereas Milnor attractors [19] attract positive measure sets that may contain no open sets.

When a dynamical system is perturbed by stationary noise, it is still of considerable interest to understand the asymptotic dynamics but there is typically no deterministic subset 
of the phase space that concisely captures all of the long term dynamics; hence a reasonable definition of an attractor will be a random set (a stationary set-valued random variable) with some notion of convergence. The notion of convergence to an attractor becomes an issue here; due to the extra structure of the random forcing there are a large number of possible ways of defining convergence to a random attractor. Our aim in this paper is to compare several different notions of convergence.

We work within the framework of random dynamical systems (RDS) to provide interpretations of the dynamics parametrized by possible 'realizations' of the noise and use tools from ergodic and probability theory. For a systematic presentation of RDS theory and its applications, we refer the reader to the monograph of L. Arnold [1].

The standard notion of convergence used for RDS was proposed by Crauel and Flandoli [14] and Schmalfuß [16] who define random attractors in terms of pullback convergence. For this one considers the limit of time going to infinity while at the same time one needs to look at the past history of the noise. The limit $t \rightarrow \infty$ then gives a compact set at time $t=0$ that depends on the past of the system; see $[14,20,22]$ for a number of results relating to the existence and structure of attractors using pullback convergence. In particular Crauel and Flandoli [14] give a method for constructing such random attractors in a very general setting by taking the intersection of pullback limits of deterministic sets. They also show how pullback convergence implies a weaker form of convergence, namely convergence in probability, which also holds forward in time. In a recent paper, Ochs [20] proposes using convergence in probability to define a random attractor, and obtains a number of results that generalize pullback results to this more general setting. The current paper examines these and other notions of convergence in detail and gives several results as to how they are related.

A motivation of our study is the following question: Given a typical initial condition and a typical noise realization, can one measure convergence in any trajectory-wise manner? Our conclusion is that there are several fundamental obstructions to doing this without excluding stochastic differential equations.

The paper is organized as follows; the remainder of this section fixes the notation we use for RDS generated by random mappings. Section 2 presents and relates a number of ways of defining convergence to a random invariant set. Section 3 looks at local and global attractors, random basins of attraction and Milnor-type random attractors. Section 4 examines an example (the van der Pol-Duffing equation forced by white noise) and explains numerically observed intermittent bursting of the two-point motion as a form of intermittency similar to that observed in the deterministic dynamics of skew-product systems. Finally, Section 5 raises a number of open questions relating to the existence of random Milnor attractors that are not merely random point attractors.

\section{Acknowledgements}

We thank Ludwig Arnold and Hans Crauel for several discussions relating to this paper. We thank the DFG and LMS for partial support of this research. We also thank our Departments at Surrey, Bremen and Exeter for hosting visits of PA and GO. 


\subsection{Random dynamical systems generated by random mappings.}

For clarity of exposition we concentrate on the discrete time case, but note that a continuous time RDS can be similarly considered via the time-one mapping defined by the flow.

Consider a metric space $(X, d(\cdot, \cdot))$ and a random mapping

$$
F: W \times X \rightarrow W \times X, \quad F(w, x)=\left(\theta w, \psi_{w}(x)\right)
$$

where $W$ is a measure space and the invertible map $\theta: W \rightarrow W$ is ergodic for the nonatomic measure $\mathcal{P}$ of a probability space $(W, \mathcal{S}, \mathcal{P}$ ) (with $\mathcal{S}$ the $\sigma$-algebra). We assume that $\psi_{w}: X \rightarrow X$ is a homeomorphism for each $w$.

We say $\theta$ is the base transformation and $\psi_{w}$ is the fiber transformation. The result of $n$ iterations of $F$ can be written as

$$
F^{n}(w, x)=\left(\theta^{n} w, \phi(n, w) x\right)
$$

where $\phi: \mathbf{Z} \times W \times X \rightarrow X$ is a cocycle over the map $\theta$ such that

$$
\begin{aligned}
\phi(n, w) x & =\psi_{\theta^{n-1} w} \circ \cdots \circ \psi_{w}(x), \\
\phi(0, w) x & =x \\
\phi(-n, w) x & =\psi_{\theta^{-n} w}^{-1} \circ \cdots \circ \psi_{\theta^{-1} w}^{-1}(x),
\end{aligned}
$$

for all $n \in\{1,2,3, \cdots\}, w \in W$ and $x \in X$. Let $d(A, B)=\sup _{a \in A} \inf _{b \in B} d(a, b)$ denote the Hausdorff semi-distance and let $h(A, B)=d(A, B)+d(B, A)$ be the Hausdorff metric on compact subsets of $X$.

A random set $A(w)$ for $w \in W$ is a subset of $X$ depending measurably on $w$ (i.e. such that the map $w \mapsto d(x, A(w))$ is measurable for all $x)$. We say a random set $A$ is forward invariant if

$$
\psi_{w} A(w) \subset A(\theta w)
$$

for almost all $w$ and it is invariant if

$$
\psi_{w} A(w)=A(\theta w)
$$

A random compact set is a random set such that $A(w)$ is compact for almost all $w$. Throughout the paper, any statements depending on $w$ apply $\mathcal{P}$-almost surely unless otherwise stated.

\section{Notions of attraction for random dynamical systems}

For a deterministic dynamical system on $X$, we say the trajectory $x(t)$ converges to an invariant set $A$ if $d(x(t), A) \rightarrow 0$ as $t \rightarrow \infty$. For the random dynamical system (1), one can only expect this to occur in a noise-dependent way, and so there are several ways to generalize convergence depending on which noise trajectories (if any) are disregarded. We present several definitions and then relate them in Theorem 2.1. 
Definition 1 Suppose that $A(w)$ is an invariant random compact subset of $X$ and $\xi(w) \in X$ is a random variable ${ }^{1}$. We say that there is almost sure pullback attraction to a random set $A$ (and write $\xi \rightarrow_{p b} A$ ) if

$$
d\left(\phi\left(n, \theta^{-n} w\right) \xi\left(\theta^{-n} w\right), A(w)\right) \rightarrow 0
$$

as $n \rightarrow \infty$ for $\mathcal{P}$-almost all $w$.

Analogously, we say $B \rightarrow_{p b} A$ for a random set $B$ if

$$
d\left(\phi\left(n, \theta^{-n} w\right) B\left(\theta^{-n} w\right), A(w)\right) \rightarrow 0
$$

as $n \rightarrow \infty$ for $\mathcal{P}$-almost all $w$. In what follows, we suppose that $A(w)$ is a compact random invariant set, $\xi(w)$ is a random variable and define

$$
r_{n}(w):=d\left(\phi(n, w) \xi(w), A\left(\theta^{n} w\right)\right)
$$

to be the distance of the trajectory starting at $\xi$ from the random invariant set $A$. Convergence of $\xi$ to $A$ then corresponds to convergence $r_{n} \rightarrow 0$ in some sense.

Definition 2 We say $\xi \rightarrow_{p r} A$ and there is (forward) attraction in probability if $r_{n} \rightarrow 0$ as $n \rightarrow \infty$ in probability.

Analogously we define $B \rightarrow_{p r} A$ for random sets $B(w)$. Recall that convergence in probability means that for any $\epsilon>0$

$$
\mathcal{P}\left(\left\{w: r_{n}>\epsilon\right\}\right) \rightarrow 0
$$

as $n \rightarrow \infty$. Note that this is equivalent to $d\left(\phi\left(n, \theta^{-n} w\right) \xi\left(\theta^{-n} w\right), A(w)\right) \rightarrow 0$ in probability due to the $\theta$ invariance of $\mathcal{P}$, see Crauel and Flandoli [14]. In particular $\xi \rightarrow_{p r} A$ follows from $\xi \rightarrow_{p b} A$. Similarly, if $B \rightarrow_{p b} A$ then $B \rightarrow_{p r} A$ and $\xi \rightarrow_{p r} A$ for any $\xi$ with $\xi(w) \in B(w)$ for almost all $w$.

This motivates the suggestion by Ochs [20] to use $\rightarrow_{p r}$ for definition of a random attractor. A stronger notion of forward convergence is the following:

Definition 3 We say $\xi \rightarrow_{f a} A$ and there is forward attraction almost surely if $r_{n}(w) \rightarrow 0$ as $n \rightarrow \infty$ for almost all $w$.

Recall that this means

$$
\mathcal{P}\left(\left\{w: r_{n}(w) \rightarrow 0 \text { as } n \rightarrow \infty\right\}\right)=1 .
$$

Note that almost sure attraction implies attraction in probability but the converse is not true. We now define two further possible trajectory-wise notions of attraction and relate all notions in Theorem 2.1.

\footnotetext{
${ }^{1}$ Recall that $\xi$ is a random variable if $w \mapsto \xi(w)$ is a $\mathcal{P}$-measurable map.
} 


\begin{tabular}{|l|l|l|}
\hline Convergence & Symbol & Meaning \\
\hline \hline forward almost sure & $\xi \rightarrow_{f a} A$ & $d\left(\phi(n, w) \xi(w), A\left(\theta^{n} w\right)\right) \rightarrow 0 w$-almost surely \\
\hline pullback & $\xi \rightarrow_{p b} A$ & $d\left(\phi\left(n, \theta^{-n} w\right) \xi\left(\theta^{-n} w\right), A(w)\right) \rightarrow 0$ almost surely \\
\hline in probability & $\xi \rightarrow_{p r} A$ & $d\left(\phi(n, w) \xi(w), A\left(\theta^{n} w\right)\right) \rightarrow 0$ in probability \\
\hline forward in mean & $\xi \rightarrow_{f m} A$ & $\frac{1}{n} \sum_{k=0}^{n-1} d\left(\phi(k, w) \xi(w), A\left(\theta^{k} w\right)\right) \rightarrow 0$ almost surely \\
\hline $\begin{array}{l}\text { forward for dense } \\
\text { subsequences }\end{array}$ & $\xi \rightarrow_{f d} A$ & $\begin{array}{l}d\left(\phi\left(n_{k}, w\right) \xi(w), A\left(\theta^{n_{k}} w\right)\right) \rightarrow 0 \text { almost surely } \\
\text { for some }\left\{n_{k}\right\} \text { with } \frac{n_{k}}{k} \rightarrow 1\end{array}$ \\
\hline in probability in the mean & $\xi \rightarrow_{p m} A$ & $\lim _{n \rightarrow \infty} \frac{1}{n} \sum_{k=0}^{n-1} \mathcal{P}\left(r_{k}(w)>\epsilon\right)=0$ for every $\epsilon>0$ \\
\hline
\end{tabular}

Table 1: The different notions of convergence we use for of a random variable $\xi$ converging to a random attractor $A$. The notion of convergence in probability in the mean is implied by all other notions and is discussed separately in Section 2.2.

Definition 4 We say $\xi \rightarrow_{f m} A$ and say there is forward attraction in the mean if $r_{n}(w)$ satisfies

$$
\frac{1}{n} \sum_{i=1}^{n} r_{i} \rightarrow 0 \text { as } n \rightarrow \infty
$$

for $\mathcal{P}$-almost all $w$, i.e. if the average of $r_{i}$ converges almost surely to zero.

Definition 5 We say $\xi \rightarrow_{f d} A$ and say there is forward attraction for dense subsequences if $r_{n}(w)$ is such that for $\mathcal{P}$-almost all $w$ there is a positive sequence $n_{k}$ with $r_{n_{k}} \rightarrow 0$ where $\frac{n_{k}}{k} \rightarrow 1$ as $k \rightarrow \infty$.

An equivalent formulation for $\xi \rightarrow_{f d} A$ is that for every $\epsilon>0$ and almost every $w$

$$
\lim _{n \rightarrow \infty} \frac{1}{n} \#\left\{0 \leq k<n: r_{k}(w) \leq \epsilon\right\}=1
$$

A summary of these notions of convergence is provided on Table 1 for convenience.

Theorem 2.1 The following implications are $(T)$ true, (F) false, or (?) unknown for the class of random dynamical system defined in Section 1.1:

\begin{tabular}{c|c|c|c|c|c|} 
& $\xi \rightarrow_{f a} A$ & $\xi \rightarrow_{p b} A$ & $\xi \rightarrow_{p r} A$ & $\xi \rightarrow_{f m} A$ & $\xi \rightarrow_{f d} A$ \\
\hline$\xi \rightarrow_{f a} A$ implies & $T$ & $F$ & $T$ & $T$ & $T$ \\
\hline$\xi \rightarrow_{p b} A$ implies & $F$ & $T$ & $T$ & $F$ & $?$ \\
\hline$\xi \rightarrow_{p r} A$ implies & $F$ & $F$ & $T$ & $F$ & $F^{2}$ \\
\hline$\xi \rightarrow_{f m} A$ implies & $F$ & $F$ & $F$ & $T$ & $T$ \\
\hline$\xi \rightarrow_{f d} A$ implies & $F$ & $F$ & $F$ & $F$ & $T$ \\
\hline
\end{tabular}

\footnotetext{
${ }^{2}$ In our counterexample (Example 8 ) the mappings $\psi_{w}$ are continuous, but unfortunately they do not satisfy the requirement to be a homeomorphism.
} 
Proof: We have already remarked that pullback convergence implies convergence in probability forward in time. It is clear from the definition that $\xi \rightarrow_{f a} A$ implies $\xi \rightarrow_{p r} A, \xi \rightarrow_{f m} A$ and $\xi \rightarrow_{f d} A$.

Now suppose $\xi \rightarrow_{f m} A$ so that for any $m>0$ there is an $N(m)$ such that

$$
\frac{1}{n} \#\left\{0 \leq i<n: r_{i}>1 / m\right\}<\frac{1}{m}
$$

for every $n \geq N(m)$. Now choose a sequence $i(k)$ such that for any $0 \leq j<i(k)$ we have $r_{j} \leq 1 / m$ for some $m$. Then $\lim _{k \rightarrow \infty} a_{i(k)}=0$ and for any $k$ with $N(m+1)>i(k) \geq N(m)$ we have

$$
\frac{k}{i(k)} \geq 1-\frac{1}{i(k)} \#\left\{0 \leq j<i(k): r_{j}>1 / m\right\}>1-\frac{1}{m}
$$

Thus $\xi \rightarrow_{f m} A$ implies that $\xi \rightarrow_{f d} A$.

The negative statements of the theorem are proved by counter-examples detailed as follows. Example 6 shows that pullback convergence does not necessarily imply convergence forward in time, and vice versa. It is also used to show that none of these notions is implied by convergence in probability. Furthermore, the same example shows that convergence for dense subsequences does not imply convergence in mean. Example 7 shows that neither convergence in probability nor the notions of almost sure convergence and pullback convergence can be inferred from convergence in mean or convergence for dense subsequences. Finally, Example 8 shows that one can have convergence in probability with neither convergence in mean nor convergence for dense subsequences.

QED

A random variable $\xi: W \rightarrow \mathbf{R}_{+}$is called tempered from above (with respect to $\theta$ ) if

$$
\limsup _{n \rightarrow \infty} \frac{1}{n} \log \xi\left(\theta^{ \pm n} w\right)=0 \quad \mathcal{P} \text {-almost surely }
$$

It is tempered from below, if $\liminf _{n \rightarrow \infty} \frac{1}{n} \log \xi\left(\theta^{ \pm n} w\right)=0$ and tempered, if it is tempered from above and from below. If $\xi$ is not tempered from above, then

$$
\limsup _{n \rightarrow \infty} \frac{1}{n} \log \xi\left(\theta^{ \pm n} w\right)=\infty
$$

almost surely. Analogously, the $\lim$ inf equals $-\infty$, if $\xi$ is not tempered from below (see e.g.[1, Prop. 4.1.3]). If we assume $\mathcal{P}$ to be non-atomic and $\theta$ ergodic then there exist nontempered random variables, see e.g. [3, Section 8]. We include a simple explicit construction of such a non-tempered variable below.

Theorem 2.2 Let $\theta: W \rightarrow W$ be an ergodic measure-preserving transformation on a nonatomic probability space $(W, \mathcal{B}, \mathcal{P})$. Then there exists a non-tempered random variable $\xi$ : $W \rightarrow \mathbf{R}$. 
Proof: Consider a partition $W=\cup_{k=1}^{\infty} W_{k}$ of $W$ into a countably infinite number of disjoint sets with positive measure. By ergodicity of $\mathcal{P}$ we have

$$
\mathcal{P}\left(\bigcup_{n=0}^{\infty} \theta^{-n} W_{k}\right)=1
$$

for each fixed $k$. Hence there exists $N_{k}$ with

$$
\mathcal{P}\left(\bigcup_{n=0}^{N_{k}} \theta^{-n} W_{k}\right) \geq 1-2^{-(k+1)}
$$

and therefore the set

$$
\hat{W}:=\bigcap_{k=1}^{\infty}\left(\bigcup_{n=0}^{N_{k}} \theta^{-n} W_{k}\right)
$$

has measure $\geq \frac{1}{2}$.

Now define $\xi(w)=\exp \left(N_{k}^{2}\right)$ for $w \in W_{k}$. For each $w \in \hat{W}$ and each $k \geq 1$ there exists $n_{k}(w) \leq N_{k}$ such that

$$
\theta^{n_{k}}(w) \in W_{k} \Rightarrow \frac{1}{n_{k}} \log \xi\left(\theta^{n_{k}} w\right)=\frac{N_{k}^{2}}{n_{k}} \geq N_{k} \geq n_{k}
$$

Since the disjointness of the $W_{k}$ implies that for fixed $w \in \hat{W}$ all the $n_{k}$ are different, it follows that

$$
\limsup _{n \rightarrow \infty} \frac{1}{n} \log \xi\left(\theta^{n} w\right)=\infty
$$

with probability $\geq \frac{1}{2}$, i.e. $\xi$ is non-tempered.

QED

Example 6 Choose any positive real-valued random variable $a(w)$ and constant $\beta$ with $0<$ $\beta<1$. For $x \in \mathbf{R}$ and $w \in W$ we set

$$
\psi_{w}(x)=\beta \frac{a(\theta w)}{a(w)} x
$$

which defines an $R D S$ over a measure preserving map $\theta: W \rightarrow W$. The corresponding cocycle can be written explicitly as

$$
\phi(n, w) x=\beta^{n} \frac{a\left(\theta^{n} w\right)}{a(w)} x .
$$

Proposition 2.3 Set $A(w)=\{0\}$ and let $\xi: W \rightarrow \mathbf{R}$ be any random variable with $\xi(w) \neq 0$ almost everywhere. Under the dynamics of (4),

(i) $\xi \rightarrow_{p r} A$, 
(ii) $\xi \rightarrow_{f d} A$,

(iii) $\xi \rightarrow_{f a} A$ if and only if a is tempered from above,

(iv) $\xi \rightarrow_{f m} A$ if and only if a is tempered from above,

(v) $\xi \rightarrow_{p b} A$ if and only if $\frac{|\xi(w)|}{a(w)}$ is tempered from above.

Proof: Statement (i) follows from

$$
\begin{gathered}
\mathcal{P}(|\phi(n, w) \xi(w)|>\epsilon) \leq \mathcal{P}\left\{w: \frac{|\xi(w)|}{a(w)}>\epsilon \beta^{-\frac{n}{2}} \text { or } a\left(\theta^{n} w\right)>\beta^{-\frac{n}{2}}\right\} \\
\leq \mathcal{P}\left\{w: \frac{|\xi(w)|}{a(w)}>\epsilon \beta^{-\frac{n}{2}}\right\}+\mathcal{P}\left\{w: a(w)>\beta^{-\frac{n}{2}}\right\} \rightarrow 0
\end{gathered}
$$

as $n \rightarrow \infty$, where we used the fact that $a(w)$ and $a\left(\theta^{n} w\right)$ have the same distribution.

To prove (ii) let $\epsilon>0$ be given. For $N \in \mathbf{N}$ define

$$
W_{N}:=\left\{w: a(w) \leq \frac{1}{N} \beta^{-N} \epsilon\right\} .
$$

By the ergodic theorem the set

$$
W^{\prime}:=\left\{w: \lim _{n \rightarrow \infty} \frac{1}{n} \#\left\{0 \leq k<n: \theta^{k} w \in W_{N}\right\}=\mathcal{P}\left(W_{N}\right) \text { for all } N\right\}
$$

has full measure. Given $w_{0} \in W^{\prime}$ and $\delta>0$ we choose $N \geq \frac{\left|\xi\left(w_{0}\right)\right|}{a\left(w_{0}\right)}$ such that $\mathcal{P}\left(W_{N}\right)>1-\delta$. Then by the definition of $r_{k}, W_{N}$ and the choice of $N$ we have

$$
\begin{gathered}
\lim _{n \rightarrow \infty} \frac{1}{n} \#\left\{0 \leq k<n: r_{k}\left(w_{0}\right) \leq \epsilon\right\}=\lim _{n \rightarrow \infty} \frac{1}{n} \#\left\{N \leq k<n: r_{k}\left(w_{0}\right) \leq \epsilon\right\} \\
\geq \lim _{n \rightarrow \infty} \frac{1}{n} \#\left\{N \leq k<n: \theta^{k} w_{0} \in W_{N}\right\}=\mathcal{P}\left(W_{N}\right)>1-\delta .
\end{gathered}
$$

Since $\delta>0$ is arbitrary, we have $\xi \rightarrow_{f d} A$.

If $a$ is tempered from above, then $\lim \sup _{n \rightarrow \infty} \frac{1}{n} \log r_{n}(w)=\log \beta<0 \mathcal{P}$-almost surely, which implies that $\xi \rightarrow_{f a} A$ and, by Theorem 2.1, $\xi \rightarrow_{f m} A$ giving one direction of (iii) and (iv). On the other hand, if $a$ is not tempered from above, then $\lim _{\sup } \rightarrow \infty \frac{1}{n} \log r_{n}(w)=\infty$. Since

$$
\frac{1}{n} \sum_{i=1}^{n} r_{i}(w) \geq \frac{1}{n} r_{n}(w) \geq \frac{1}{n} \log r_{n}(w)
$$

there is no convergence in mean nor forward almost sure, proving the other direction of (iii) and (iv).

To prove $(\mathrm{v})$ note that

$$
\frac{1}{n} \log r_{n}\left(\theta^{-n} w\right)=\log \beta+\frac{1}{n} \log a(w)+\frac{1}{n} \log \frac{\left|\xi\left(\theta^{-n} w\right)\right|}{a\left(\theta^{-n} w\right)}
$$


where the $\lim$ sup equals $\log \beta$ almost surely if $\frac{|\xi(w)|}{a(w)}$ is tempered from above and is infinite otherwise.

QED

Summarizing the dynamics of Example 6 with $0<\beta<1$ and an arbitrary $\theta$ preserving an ergodic and non-atomic probability measure, we have:

- If $a$ is tempered then $\xi \rightarrow_{p r} A, \xi \rightarrow_{f d} A, \xi \rightarrow_{f a} A$ and $\xi \rightarrow_{f m} A$ for arbitrary random variables $\xi$, and $\xi \rightarrow_{p b} A$ if $|\xi|$ is tempered from above.

- If $a$ is tempered from below but not from above then $\xi \rightarrow_{p r} A$ and $\xi \rightarrow_{f d} A$, but neither $\xi \rightarrow_{f a} A$ nor $\xi \rightarrow_{f m} A$ if $\xi$ is non-zero. Furthermore, $\xi \rightarrow_{p b} A$ if $|\xi|$ is tempered from above.

- If $a$ is tempered from above but not from below then $\xi \rightarrow_{p r} A, \xi \rightarrow_{f d} A, \xi \rightarrow_{f a} A$ and $\xi \rightarrow_{f m} A$ for arbitrary random variables $\xi$. However, $\xi \not \rightarrow_{p b} A$ even for constant random variables $\xi$ that are non-zero.

- If $a$ is neither tempered from above nor from below then still $\xi \rightarrow_{p r} A$ and $\xi \rightarrow_{f d} A$ for arbitrary random variables $\xi$, but neither $\xi \rightarrow_{f a} A$, nor $\xi \rightarrow_{f m} A$ (if $\xi$ is non-zero), nor $\xi \rightarrow_{p b} A$ for "most" (e.g. constant) $\xi$.

Example 7 Let $W=\{0\}$ be a one point set and $X:=S^{1} \times[0, \infty)$ be viewed as a subset of $\mathbf{R}^{3}$ with the induced metric. Define (with $0 \leq \alpha<1$ )

$$
\psi_{0}: X \rightarrow X, \quad \psi_{0}\left(e^{2 \pi i \alpha}, y\right)=\left(e^{2 \pi i(\sqrt{\alpha}+y)}, g(y)\right)
$$

where $g(y)=\frac{y}{y+1}$ for $0 \leq y<1$ and $g(y)=\frac{y}{2}$ for $y \geq 1$. This defines an $R D S$ over the trivial base system $\theta(0)=0$. With $A=\left\{\left(e^{0}, 0\right)\right\}$ it can be shown that $\xi \rightarrow_{f d} A$ and $\xi \rightarrow_{f m} A$ for arbitrary initial values $\xi(w)=\left(e^{2 \pi i \alpha}, y\right)$.

However, if $y>0$ then $\phi(n, 0) \xi(0) \nrightarrow \rightarrow(1,0)$. Hence there is no almost sure convergence, which for the one point probability space $\{0\}$ is equivalent to convergence in probability.

Another non-random example (that is moreover smooth) exhibiting such convergence is the time-one map of a flow with an asymptotically stable homoclinic cycle.

Next we give an example which shows that convergence in probability does not necessarily imply convergence for dense subsequences.

Example 8 Let $W=(0,1]^{\mathbf{Z}}$ be endowed with the infinite product $\mathcal{P}$ of Lebesgue measure on the unit interval $(0,1]$ and $\theta$ be the $\mathcal{P}$-preserving left shift on $W$ given by $(\theta w)_{n}=w_{n+1}$. Let

$$
X:=\mathbf{Z}^{+} \cup\left\{\frac{1}{k+1}: k \in \mathbf{N}\right\}=\left\{0, \ldots, \frac{1}{n+1}, \frac{1}{n}, \ldots, \frac{1}{2}, 1,2,3, \ldots\right\},
$$

with metric $d(x, y)=|x-y|$ induced from $\mathbf{R}$. Then 0 is the only accumulation point in $X$ and hence a mapping from $X$ to itself is continuous if and only if it is continuous in 0. 
We define a random dynamical system as follows. Choose a sequence $\left\{a_{k}\right\}_{k \geq 2} \subset(0,1)$ with the properties

$$
a_{k} \downarrow 0, \quad \sum_{k=2}^{\infty} a_{k}=\infty \quad \text { and } \lim _{N \rightarrow \infty} \sum_{\alpha N \leq k \leq \beta N} a_{k}=0
$$

for any $0<\alpha<\beta$. This holds for instance with $a_{k}=\frac{1}{k \log k}$ since the function $\frac{1}{x \log x}$ has antiderivative $\log \log x$ and

$$
\sum_{\alpha N \leq k \leq \beta N} a_{k} \leq \frac{1}{\alpha N \log \alpha N}+\int_{\alpha N}^{\beta N} \frac{d x}{x \log x} \leq \frac{1}{\alpha N \log \alpha N}+\log \left(\frac{\log N+\log \beta}{\log N+\log \alpha}\right) .
$$

Now define $\psi_{w}(0)=0, \psi_{w}(1)=\frac{1}{2}$,

$$
\psi_{w}\left(\frac{1}{k}\right)=\left\{\begin{array}{cl}
\frac{1}{k+1}, & \text { if } w_{0}>a_{k}, \\
k+1, & \text { if } w_{0} \leq a_{k}
\end{array}\right.
$$

for $k \geq 2, \psi_{w}(k)=k+1$ if $k \geq 3$ is odd, and

$$
\psi_{w}(2 k)=\left\{\begin{array}{cl}
2 k+1, & \text { if } w_{-k}>a_{k}, \\
\frac{1}{2 k+1}, & \text { if } w_{-k} \leq a_{k}
\end{array}\right.
$$

for $k \geq 1$, where $w=\left\{w_{n}\right\}_{n \in \mathbf{Z}} \in W$.

We note that the mapping $\psi_{w}: X \rightarrow X$ is continuous, since for any $w$ it maps $\frac{1}{k}$ to $\frac{1}{k+1}$ for all sufficiently large $k$, but it is not a homeomorphism.

Proposition 2.4 For the $R D S$ defined in Example 8, any non-zero random variable $\xi$ : $W \rightarrow X$ and $A=\{0\}$ satisfies

$$
\xi \rightarrow_{p r} A \quad \text { and } \quad \xi \nrightarrow_{f d} A
$$

Proof: $\quad$ To see that $\xi \rightarrow_{p r} 0$, consider for fixed $w$ an orbit with initial condition $\frac{1}{k}, k \geq 2$. Then $\phi(n, w) \frac{1}{k}$ is either $\frac{1}{k+n}$ or $k+n$. In the latter case the orbit will return to $\left\{\frac{1}{l}: l \in \mathbf{N}\right\}$ after at most $k+n$ more iteration steps. Hence $\phi(n, w) \frac{1}{k}=\frac{1}{k+n}$ whenever $w_{l}>a_{k+l}$ for $\frac{n-k-1}{2} \leq l<n$. This is clearly the case if $n \geq k$ and $w_{j-k}>a_{j}$ for $\frac{n}{2} \leq j \leq 2 n$. If now $\xi(w)=\frac{1}{k(w)}$ is a random variable and $\epsilon>0$ is given, choose $k_{0}$ with $\mathcal{P}\left(k(w) \leq k_{0}\right)>1-\frac{\epsilon}{2}$. Then, if $n \geq k_{0}$,

$$
\mathcal{P}\left(\phi(n, w) \xi(w)>\frac{1}{n}\right)<\frac{\epsilon}{2}+\sum_{n / 2 \leq j \leq 2 n} a_{j} .
$$

The last term tends to 0 as $n \rightarrow \infty$, which shows $\xi \rightarrow_{p r} 0$.

It remains to consider the case $\xi(w) \geq 1$. In order to conclude $\xi \rightarrow_{p r} 0$ for arbitrary random variables $\xi: W \rightarrow X$ we have to show that for $k \geq 1$ there exists with probability one an $n>0$ with $\phi(n, w) k<1$ (see Proposition 2.5 below). This is clear for $k=1$ since $\phi(1, w) 1=\frac{1}{2}$. For $k>1$ we have $\phi(n+1, w) k=\frac{1}{k+n+1}$ if for the first time $k+n$ is even 
and $w_{\frac{n-k}{2}} \leq a_{\frac{n+k}{2}}$. Since the sum over all $a_{\frac{n+k}{2}}$ for $n+k$ even is unbounded, this occurs with probability 1 for some $n$ by the Borel-Cantelli lemma.

However, in general there is no convergence for dense subsequences. Consider, for instance, an orbit with initial value 1. By analogy to the previous considerations, if $w_{l-1}>a_{l}$ for $\frac{n}{2} \leq l<n$ and $w_{n-1} \leq a_{n}$, then $\phi(n-1, w) 1=\frac{1}{n}, \phi(l-1, w) 1=l$ for $n<l \leq 2 n$, and $\phi(2 n, w) 1=\frac{1}{2 n+1}$.

This happens with probability greater than $a_{n} b_{n}$, where

$$
b_{n}=1-\sum_{n / 2 \leq l<n} a_{l} \rightarrow 1 \text { as } n \rightarrow \infty .
$$

Hence $\sum_{n=2}^{\infty} a_{n} b_{n}=\infty$, which shows with the help of the Borel-Cantelli lemma that with probability 1 there exist infinitely many $n$ with $\phi(l, w) 1 \geq 1$ for $n<l \leq 2 n$, i.e.

$$
\limsup _{n \rightarrow \infty} \frac{1}{n} \#\{0 \leq k<n: \phi(k, w) 1 \geq 1\} \geq \frac{1}{2}
$$

and therefore $\xi \dashv_{f d}\{0\}$.

QED

Proposition 2.5 Let $A$ be an invariant random compact set and $B$ be a random set with $A \subset B$. Assume $\xi \rightarrow_{p r} A$ for every random variable $\xi$ with $\xi(w) \in B(w)$ almost surely. Define $C(w)$ to be the set of all $x$ such that $\phi(n, w) x \in B\left(\theta^{n} w\right)$ for some $n \geq 0$.

Then $\xi \rightarrow_{p r}$ A for every random variable $\xi$ such that $\xi(w) \in C(w)$ almost surely.

Proof: $\quad$ Choose a random variable $n(w) \geq 0$ such that $\phi(n(w), w) \xi(w) \in B\left(\theta^{n} w\right)$. For $m \in \mathbf{N}$ define $W_{m}=\{w \in W: n(w)=m\}$. Let $\epsilon>0$ be given and choose $M \in \mathbf{N}$ with $\mathcal{P}(n(w)>M)<\frac{\epsilon}{2}$. Choose random variables $\zeta_{0}, \ldots, \zeta_{M}$ with $\zeta_{m}(w)=\xi(w)$ if $w \in W_{m}$ and $\zeta_{m}(w) \in A(w)$ if $w \notin W_{m}$, i.e. $\phi(m, w) \zeta_{m}(w) \in B\left(\theta^{m} w\right)$ for all $m, w$. By assumption there exist $N_{0}, \ldots, N_{M}>0$ such that

$$
\mathcal{P}\left(d\left(\phi(n+m, w) \zeta_{m}(w), A\left(\theta^{n+m} w\right)\right) \geq \epsilon\right)<\frac{\epsilon}{2(M+1)}
$$

for all $m$ and $n \geq N_{m}$. Since

$$
\begin{gathered}
\left\{w: d\left(\phi(n, w) \xi(w), A\left(\theta^{n} w\right)\right) \geq \epsilon\right\} \subset \\
\bigcup_{m=0}^{M}\left\{w \in W_{n}: d\left(\phi(n, w) \zeta_{m}(w), A\left(\theta^{n} w\right)\right) \geq \epsilon\right\} \cup\{w: N(w)>M\},
\end{gathered}
$$

this implies that

$$
\mathcal{P}\left\{w: d\left(\phi(n, w) \xi(w), A\left(\theta^{n} w\right)\right) \geq \epsilon\right\}<\epsilon
$$

whenever $n \geq N(\epsilon)=\max _{m=0, \ldots, M} N_{m}+m$.

In general, convergence in probability and convergence for dense subsequences are not implied by each other. Under additional assumptions there is however a connection; for 
example, if there exists a compact set $N(w)$ such that $A(w)$ is contained in the interior of $N(w)$ and $N \rightarrow_{p b} A$ (i.e. if $A$ is a set attractor in the pullback sense) then

$$
B(w)=\left\{x \in X: x \rightarrow_{f d} A\right\}
$$

is a basin of $A$ for convergence in probability [20, Thms. 8 and 10], that is, $\xi \rightarrow_{p r} A$ for every random variable $\xi$ with $\xi(w) \in B(w)$ almost surely (cf. Section 3.1).

Finally we discuss a condition under which convergence for dense subsequences does imply convergence in the mean.

Theorem 2.6 If $r_{n}(w)$ is almost surely bounded then $\xi \rightarrow_{f d} A$ if and only if $\xi \rightarrow_{f m} A$.

Proof: One direction of the implication is shown in Theorem 2.1. Now assume that there is attraction for dense subsequences and choose $M$ with $r_{n}(w) \leq M$ for all $n$ and almost all $w$. For all $\epsilon>0$ there is a $K$ such that $r_{n_{k}}<\epsilon$ and $\frac{n_{k}}{n}>1-\epsilon$ for all $k>K$. Thus, if $N>n_{K}$ we have

$$
\begin{aligned}
& \frac{1}{N} \sum_{i=1}^{N} r_{i}<\frac{1}{N}\left(n_{K} M+\sum_{n_{K}+1}^{N} r_{i}\right) \\
& \leq \frac{1}{N}\left(n_{K} M+(1-\epsilon) N \epsilon+\epsilon N M\right)
\end{aligned}
$$

and so

$$
\frac{1}{N} \sum_{i=1}^{N} r_{i}<\frac{n_{K} M}{N}+\epsilon(1+M)
$$

Taking $N$ arbitrarily large and $\epsilon$ arbitrarily small, we observe that there is attraction in the mean.

QED

Theorem 2.6 does not hold unless $r_{n}(w)$ is bounded (see also Example 6). One can choose sequences of variables (that are not even random) such as

$$
r_{n}= \begin{cases}n & \text { if } n=k ! \text { for some } k \\ 0 & \text { otherwise }\end{cases}
$$

Such choices of $r_{n}$ tend to zero on dense subsequences but not in the mean. This can easily be adapted so that the lim sup or even the lim inf of the mean becomes unbounded.

The previous example can also be used as an example of a sequence that does not converge to zero in the sense of probability but which does converge for dense subsequences. It can be adapted to converge in mean by modifying to allow $r_{n}$ to take values 0 or 1 .

\subsection{Observability of random attractors}

Attraction in probability does not imply that typical trajectories will converge to the attractor. In fact there seem to be fundamental obstructions to reformulating attraction to a random attractor in a pathwise sense, in that neither attraction on average nor dense subsequences need to occur for almost all paths converging to an attractor. However, one can obtain convergence in some 'random' norm as follows. 
Proposition 2.7 If $r_{n}(w) \rightarrow_{p r} 0$ then there a sequence of random variables $\eta_{n}(w)$ with $\eta_{n} \rightarrow_{p r} 1$ such that

$$
\eta_{n}(w) r_{n}(w) \rightarrow 0
$$

as $n \rightarrow \infty$ for all $w \in W$.

Proof: Consider such an $r_{n}(w)$ converging in probability. We can find a decreasing sequence $\epsilon_{n}>0$ such that $\epsilon_{n} \rightarrow 0$ and $P\left(\left\{w: r_{n}>\epsilon_{n}\right\}\right) \rightarrow 0$ as $n \rightarrow \infty$. Now define

$$
\eta_{n}(w)=\left\{\begin{aligned}
1 & \text { if } r_{n}(w) \leq \epsilon_{n} \\
\frac{\epsilon_{n}}{r_{n}(w)} & \text { if } r_{n}(w)>\epsilon_{n}
\end{aligned}\right.
$$

Note that $\eta_{n}(w) \rightarrow_{p r} 1$ and moreover

$$
r_{n}(w) \eta_{n}(w) \leq \epsilon_{n}
$$

for all $n$ and $w$; hence the result follows.

QED

\subsection{Forward convergence and invariant measures}

An invariant measure for the RDS (1) is a probability measure $\mu$ on $W \times X$ that is invariant under $F$, and whose marginal on $W$ coincides with the given measure $\mathcal{P}$. Equivalently, via a disintegration, $\mu$ is given by a family of probabilities $\left(\mu_{w}\right)_{w \in W}$ on $X$ with the invariance condition $\psi_{w} \mu_{w}=\mu_{\theta w}$.

Any (forward) invariant random compact set supports at least one invariant measure, see for example [1, Theorem 1.6.13]. It is known that if $A$ is a global attractor in probability, then $A$ supports all invariant measures of the system [20, Theorem 2]. However, in Example 7 the only invariant measure is the point mass in $(1,0)$, i.e. in general the support of all invariant measures does not attract in the sense of convergence in probability. In this example we have convergence for dense subsequences to $(1,0)$, instead. By contrast, the only invariant probability in Example 8 is the point mass in 0 which attracts in probability, but not for dense subsequences. Thus none of our notions of attraction is in general related to the support of invariant measures. In order to get an appropriate notion, we define a weaker notion of convergence:

Definition 9 Let $A$ be an invariant random compact set and $\xi(w)$ a random variable. We say that $A$ attracts $\xi$ in probability in the mean (and we write $\xi \rightarrow_{p m} A$ ), if

$$
\lim _{n \rightarrow \infty} \frac{1}{n} \sum_{k=0}^{n-1} \mathcal{P}\left(r_{k}(w)>\epsilon\right)=0
$$

for every $\epsilon>0$.

This is implied by both convergence in probability and convergence for dense subsequences and hence by any type of convergence discussed previously. Examples 7 and 8 show that it is strictly weaker than any other notion. 


\section{Theorem 2.8}

(i) Let the state space $X$ be separable, $A$ be an invariant random compact set and $B$ a forward invariant random set with $A \subset B$ such that $\xi \rightarrow_{p m} A$ for every random variable $\xi$ with $\xi(w) \in B(w)$. Then $\mu(A)=1$ for every invariant probability measure with $\mu(B)=1$.

(ii) Assume that $X$ is locally compact and the system possesses a global random attractor, i.e. a compact invariant random set $A$ with $\xi \rightarrow_{p r} A$ for every random variable $\xi$. Let $S$ be a random compact set which supports all invariant measures (i.e. $\mu(S)=1$ for every invariant probability $\mu$ ). Then $\xi \rightarrow_{p m} S$ for every random variable $\xi$.

Proof: Part (i) is proved in [20, Theorem 3]. To prove (ii) assume that $\xi\lrcorner_{p m} S$ for some random variable $\xi$. Consider the empirical measures

$$
\mu_{n}=\frac{1}{n} \sum_{k=0}^{n-1} F^{k} \nu,
$$

where $\nu$ is given by its disintegration $\nu_{w}=\delta_{\xi(w)}$. By assumption, the closed $\epsilon$ neighbourhood $A_{\epsilon}(w)$ of $A(w)$ defines a random compact set with $\lim _{n \rightarrow \infty} \mu_{n}\left(A_{\epsilon}\right)=1$. From Crauel [12, Theorem 4.3] it follows that the sequence $\left\{\mu_{n}\right\}$ is tight, i.e. every subsequence has an accumulation point with respect to weak convergence which is an invariant measure by $[1$, Theorem 1.5.8].

If $\xi \dashv_{p m} S$ then there exists an $\epsilon>0$ and a subsequence $\mu_{n(k)}$ with $\mu_{n(k)}\left(S_{\epsilon}\right) \leq 1-\epsilon$ for all $k$. This implies that $\mu(S) \leq 1-\epsilon$ for any accumulation point $\mu$ of $\left\{\mu_{n(k)}\right\}$, contradicting the assumption on $S$.

QED

The assumptions of Part (ii) are in particular fulfilled if the state space $X$ is compact. In this case the union of the supports of all invariant measures attracts in probability in the mean.

\section{Global attractors and Milnor attractors}

For deterministic dynamics, a global attractor is a compact invariant set that attracts all initial conditions. A local attractor is a subset of a global attractor that only attracts a subset of initial conditions, its basin of attraction. Typically a global attractor will decompose into several local attractors, but will also include unstable (repelling) invariant sets, saddles and connecting orbits. These local attractors in the asymptotically stable sense, may (for compact finite-dimensional attractors) be decomposed into smaller Milnor attractors in the sense of [19].

We discuss possible definitions for local and Milnor-type attractors for RDS; first we review the standard concept of a global attractor in this setting. Let $K(w)$ and $B(w)$ be random sets. We say $K$ absorbs $B$ if $\phi\left(n, \theta^{-n} w\right) B\left(\theta^{-n} w\right) \subset K(w)$ for for almost all $w$ and 
large enough $n$ (which may depend on $w$ ). Now suppose that $K$ absorbs $B$ and that $K$ is compact; Crauel and Flandoli [14] show that one can construct a random invariant set

$$
\Omega_{B}(w)=\bigcap_{N>0} \overline{\bigcup_{n>N} \phi\left(n, \theta^{-n} w\right) B}
$$

such that $B \rightarrow_{p b} \Omega_{B}$. By taking the closure of the union of $\Omega_{B}(w)$ for all bounded nonrandom sets $A$ one can construct a global attractor.

\subsection{Random basins of attraction}

As proposed in [6], in analogy with Milnor attractors [19] for the skew product system one can define a random basin of attraction for a compact random invariant set $A(w)$ as follows. We say $A(w)$ has a random basin of forward attraction $\mathcal{B}(A)$ where

$$
\mathcal{B}(A)(w)=\left\{x \in X: d\left(\phi(n, w) x, A\left(\theta^{n} w\right)\right) \rightarrow 0 \text { as } n \rightarrow \infty\right\} .
$$

Clearly $A(w) \subset \mathcal{B}(A)(w)$ for almost every $w$, as $A$ is invariant.

If some reference measure $\ell$ on $X$ is given that assigns positive measure to all open sets (e.g. the Lebesgue measure in the case $X=\mathbf{R}^{d}$ ) we can, following [6], define $A(w)$ to be a random forward almost sure (fa) Milnor attractor (with respect to $\ell$ ) if $\ell(\mathcal{B}(A)(w))>0$ for $\mathcal{P}$-almost all $w$, and if any smaller compact random invariant set $A^{\prime}(w) \subsetneq A(w)$ has $\ell\left(\mathcal{B}(A)(w) \backslash \mathcal{B}\left(A^{\prime}\right)(w)\right)>0$. However, this definition is often too restrictive; it may be that the basin has no measure even if all points in $X$ converge to some $A(w)$ in the sense of probability. We therefore propose a weaker definition of basin of attraction.

We say that a random set $B$ is a random basin of (forward) attraction in probability of an invariant random compact set $A$, if

(a) $B$ is forward invariant,

(b) $A \subset B$, and

(c) for every random variable $\xi$ with $\xi(w) \in B(w)$ we have $\xi \rightarrow_{p r} A$,

where containments are understood to be almost sure. Clearly the basin of forward attraction is contained in a basin of attraction in probability. Given a random invariant compact $A(w)$ we say it is a global random attractor in probability if $B(w)=X$ is a basin of attraction in probability of $A$. We say $A$ a local attractor if there exists a basin $B$ such that (almost surely) $B(w)$ contains an open neighbourhood of $A(w)$. We say $A$ is a forward in probability Milnor attractor if there is a random basin $B(w)$ such that $\ell(B(w))>0$ for almost all $w$ and is a global Milnor attractor if $\ell(X \backslash B(w))=0$ for almost all $w$.

In the following we restrict our attention to the case $X=\mathbf{R}^{d}$. If $A(w)=\{x(w)\}$ is a single point, then $\mu_{w}=\delta_{x(w)}$ is an invariant measure. If the $\psi_{w}$ are smooth, then there exists a local stable manifold (see [1, Chapter 7.5]), which is a basin of forward attraction for $A$. In particular, if the top Lyapunov exponent (LEs) of the linearized system at $x(w)$ is negative, then the stable manifold contains an open neighbourhood of $x(w)$, i.e. $A$ is a local attractor in the sense of almost sure convergence forward in time and thus also in probability. 
Let the mappings $\psi_{w}, \psi_{\theta w}, \psi_{\theta^{2} w}, \ldots$ be stochastically independent (which is for example the case if $\psi$ is the time- 1 map of the solution operator of a stochastic differential equation). Then $\psi_{w}$ generates a Markov process with transition probabilities

$$
P(x, B)=\mathcal{P}\left\{w: \psi_{w}(x) \in B\right\} .
$$

If in addition the $\psi_{w}$ are smooth then we have the following result:

Theorem 3.1 Assume the Markov process has a unique invariant probability measure $\rho$ which is equivalent to Lebesgue measure and has full support. Further suppose that all Lyapunov exponents of $\rho$ are negative.

Then $\mu_{w}=\lim _{n \rightarrow \infty} \phi\left(n, \theta^{-n} w\right) \rho$ (in the sense of weak convergence) is supported by a finite set $A(w)$ and defines an invariant measure for the $R D S$ generated by $\psi$.

The random set $A$ is a global Milnor attractor of $\phi$ with basin $\mathcal{B}(A)(w)$ such that $\xi \rightarrow_{f a} A$ for every $\xi$ with $\xi(w) \in \mathcal{B}(A)(w)$.

Proof: The measure $\mu$ is invariant for $\phi$ by [1, Theorem 1.7.2]. It has finite support $A(w)$ by [11, Prop. 2.1]. The invariance of $\mu$ implies that $A$ is an invariant random compact set. The uniqueness of $\rho$ implies that $\rho$ is ergodic and hence $\mu$ is an ergodic measure for the skew product $F$ (cf. [1, Theorem 1.7.2]). Therefore $A$ cannot be decomposed into smaller invariant random sets. It remains to show that for almost every $w$ the set

$$
\mathcal{B}(w)=\mathcal{B}(A)(w)=\left\{x: d\left(\phi(n, w) x, A\left(\theta^{n} w\right)\right) \rightarrow 0\right\}
$$

has full Lebesgue measure. We know that $\mathcal{B}$ contains the local stable manifold $M^{s}(w)$ which is an open neighbourhood of $A(w)$. This implies that for almost all $w$

$$
\begin{gathered}
\liminf _{n \rightarrow \infty} \rho\left\{x: \phi\left(n, \theta^{-n} w\right) x \in M^{s}(w)\right\}=\liminf _{n \rightarrow \infty} \rho\left(\phi\left(n, \theta^{-n} w\right)^{-1} M^{s}(w)\right) \\
=\liminf _{n \rightarrow \infty}\left(\phi\left(n, \theta^{-n} w\right) \rho\right)\left(M^{s}(w)\right) \geq\left(\lim _{n \rightarrow \infty} \phi\left(n, \theta^{-n} w\right) \rho\right)(A(w))=\mu_{w}(A(w))=1,
\end{gathered}
$$

Since $\rho$ is a probability measure it follows

$$
\lim _{n \rightarrow \infty} \rho\left\{x: \phi\left(n, \theta^{-n} w\right) x \in M^{s}(w)\right\}=1 \quad \mathcal{P} \text {-almost surely }
$$

Considering the product measure $\mathcal{P} \times \rho$ defined on $W \times X$ and using the invariance of $\mathcal{P}$ under $\theta$ we obtain

$$
\begin{aligned}
1 & =\lim _{n \rightarrow \infty}(\mathcal{P} \times \rho)\left\{(w, x): \phi\left(n, \theta^{-n} w\right) x \in M^{s}(w)\right\} \\
& =\lim _{n \rightarrow \infty}(\mathcal{P} \times \rho)\left\{(w, x): \phi\left(n, \theta^{n} w\right) x \in M^{s}\left(\theta^{n} w\right)\right\}
\end{aligned}
$$

Since

$$
\begin{aligned}
& \left\{(w, x): \phi\left(n, \theta^{n} w\right) x \in M^{s}\left(\theta^{n} w\right)\right\} \\
& \subset\left\{(w, x): \lim _{k \rightarrow \infty} d\left(\phi(k, w) x, A\left(\theta^{k} w\right)\right)=0\right\}=\{(w, x): x \in \mathcal{B}(w)\},
\end{aligned}
$$

this shows that $(\mathcal{P} \times \rho)\{(w, x): x \in \mathcal{B}(w)\}=1$ and hence $\rho\{x: x \in \mathcal{B}(w)\}=1$ for almost all $w$. This finishes the proof because $\rho$ is equivalent to Lebesgue measure.

QED 


\section{Example: stochastic van der Pol-Duffing equation}

In this section we consider the well-known van der Pol-Duffing oscillator with multiplicative stochastic forcing. This is given by the Itô stochastic differential equation

$$
\begin{aligned}
d x & =y d t \\
d y & =\left(\mu x+\alpha y+a x^{3}+b x^{2} y\right) d t+\sigma x d W_{t}
\end{aligned}
$$

where $\mu, \alpha, a, b$ and $\sigma$ are parameters (one can scale $|a|=|b|=1$ by coordinate transformations), $W_{t}$ is a standard one-dimensional Brownian motion ( $\sigma$ is the noise strength). The dynamics in the absence of noise is that of the normal form for the Bogdanov-Takens bifurcation when Hopf $(\alpha=0)$ and pitchfork $(\mu=0)$ bifurcations meet. A summary of the noise-free $(\sigma=0)$ dynamics for $\mu, \alpha$ small can be found in [17] and of the noise-forced system in [1, Chapter 9.4].

The equation (5) has been examined from the point of random dynamical systems by a number of authors in recent years. A stochastic Hopf bifurcation in this system has been examined in detail in [5, 9]. Schenk-Hoppé [22] and Keller and Ochs [18] have examined numerical approximations of random attractors that are respectively ensemble and set-based. For the parameters regions examined (where the origin is not the unique random attractor), the conclusion of these papers was that (a) the random attractors are nontrivial random sets with a lot of fine scale structure and (b) almost all points in the ensemble are attracted to an invariant measure consisting of two random delta functions. By Theorem 3.1 the support of these delta functions is a global random Milnor attractor. This random Minor attractor is embedded in a much larger random set attractor such that some random invariant measures supported there have positive top LEs.

Here we give some numerical evidence that in a different parameter regime there exist random Milnor attractors with positive top LEs that are not just random point attractors. This compares with the appearance of non-point random Milnor attractors as observed in analogue electronic simulations with pseudo-random noise in [7]. Similar random attractors have been observed in the Kramers/Duffing oscillator by [23, 18] and analytically in [10].

The system (5) was simulated using a Euler-Nakayama integrator with time step $h=0.01$ and the standard $\mathrm{C}$ pseudorandom number generator 'drand48'. The results suggest that this system has a random Milnor attractor with positive top LEs for a range of parameter values. Fixing $a=-1, b=-1$ and $\sigma=0.5$ we show in Figure 1 the values of $(\alpha, \mu)$ where we find positive top LEs (these LEs are approximations over 5000 time units). This occurs for a 'wedge' of parameters, including the region where the system for $\sigma=0$ has bistable fixed point and limit cycle attractors. The prediction of positive top LEs becomes unreliable for this number of time units near $\alpha=\mu=0$, but examination of the convergence suggests that it is usually reliable when the parameters are of order 1.

We now examine in more detail the random attractor for the parameter values

$$
\alpha=1.4, \quad \mu=1, \quad a=-1, \quad b=-1, \quad \sigma=0.5
$$

the stochastic differential equation (5) giving a positive top LE (approximately 0.042). Figure 2 shows the evolution of a grid of uniformly distributed initial conditions at time 


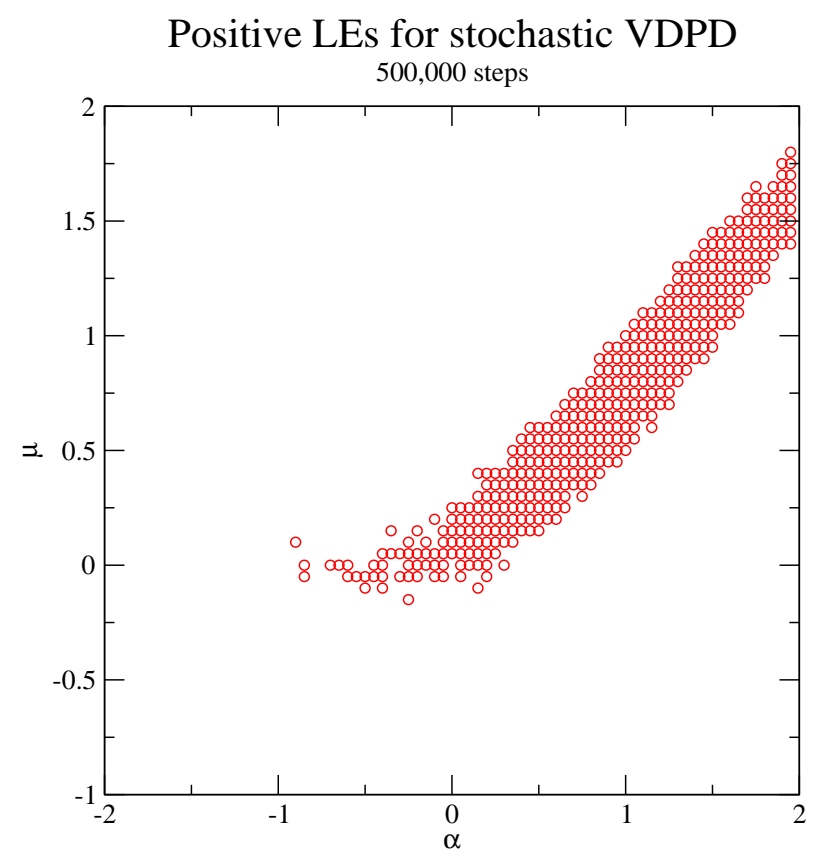

Figure 1: The circles indicate locations where random attractors with positive top Lyapunov exponents for (5) found as a function of $(\alpha, \mu)$. 500,000 time steps were used to compute the Lyapunov exponent starting at a randomly chosen initial point. The other parameters were set to $a=b=-1, \sigma=0.5$. The attractors with positive top Lyapunov exponents are located near a line of homoclinic bifurcations for the noise-free $(\sigma=0)$ system where there is bistability between attracting equilibria and a limit cycle. 

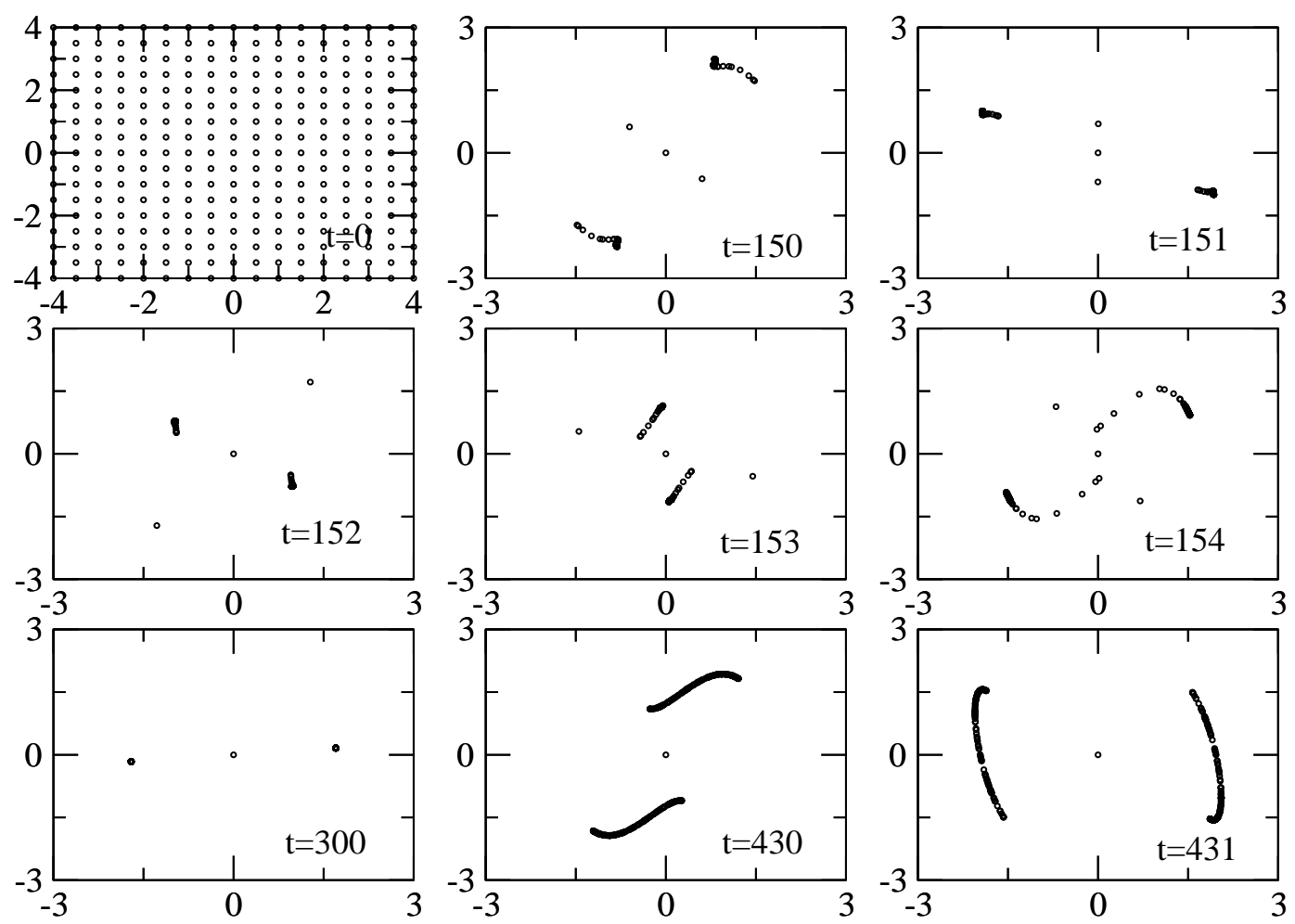

Figure 2: This figure shows the evolution of a cloud of uniformly distributed initial points at $t=0$ at various times in the future, for the van der Pol-Duffing oscillator (5) with a fixed realization of the noise and parameters $\alpha=1.4, \mu=1, \sigma=0.5$ and $a=b=-1$. Observe the presence of a random attractor that changes size considerably and intermittently; at $t=300$ it appears to have collapsed but it intermittently expands to a much larger set at later times. 


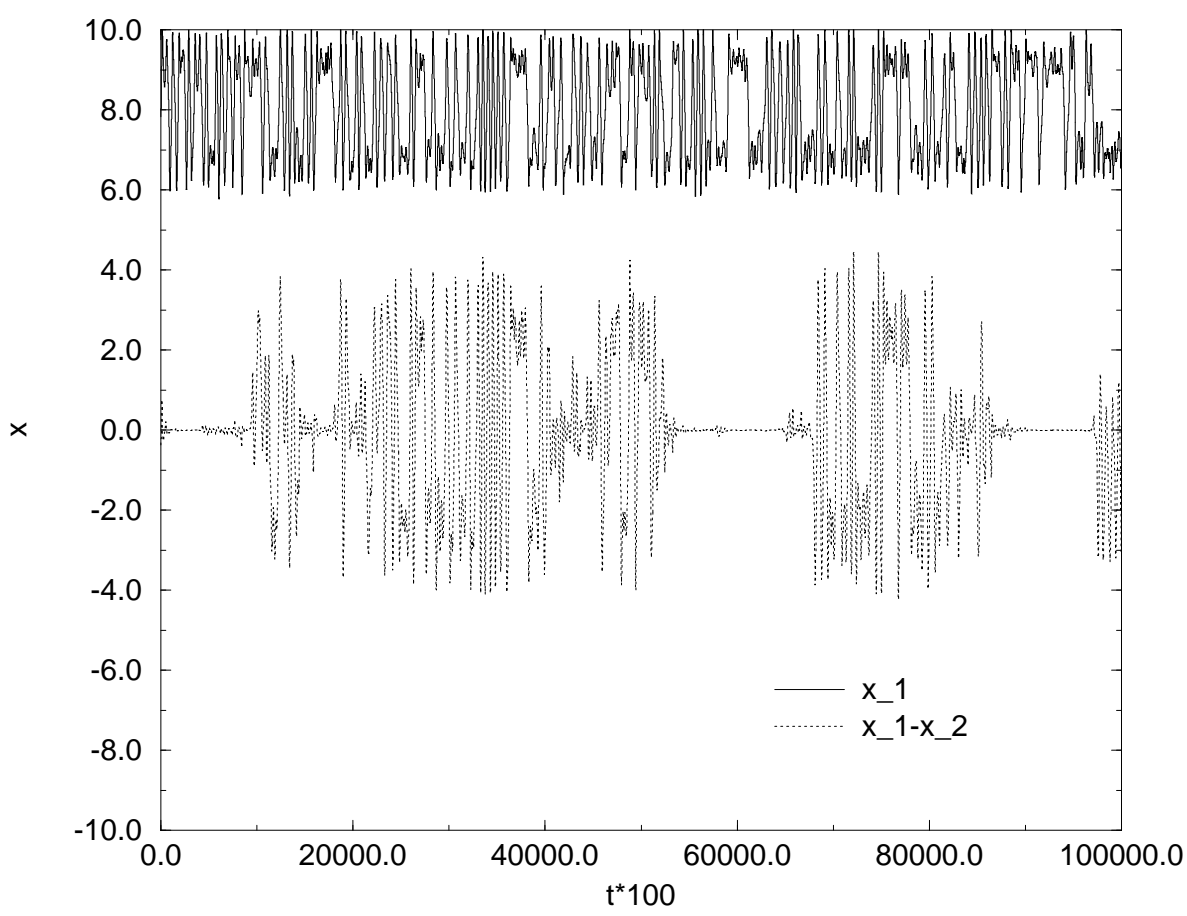

Figure 3: The top trace shows $x_{1}$ as a function of time, displaced for clarity (parameters are as in Figure 2). The bottom trace shows the difference between two trajectories $x_{1}(t)$ and $x_{2}(t)$ of the van der Pol-Duffing equation using the same noise realization but different initial conditions. The intermittent periods where the trajectories are near identical correspond to phases where the Milnor attractor is small. These are interspersed with bursts where the two trajectories separate widely.

$t=0$. The initial conditions converge towards a low dimensional random attractor $A(w)$ that evolves with the flow as illustrated.

The dynamics is highly intermittent; repeatedly (for example at $t=300$ for this realization of the noise) the attractor appears to have collapsed onto a pair of random fixed points. However, it expands out again to an attractor with size of order 1 (for example at $t=430$ in Figure 2). To examine this in more detail, we consider two trajectories of the noise-forced system $(5),\left(x_{1}(t), y_{1}(t)\right)$ and $\left(x_{2}(t), y_{2}(t)\right)$ subject to the same realization of the noise. Figure 3 shows a plot of $x_{1}$ (displaced upwards for clarity) and the difference $x_{1}-x_{2}$ against time. Observe the presence of 'laminar' phases where the difference decays almost to zero. This is consistent with the top LE being positive but the convergence being non-uniform and is analogous to on-off intermittency for the difference $x_{1}-x_{2}$ (see $[21,7]$ ). 


\section{Discussion}

In conclusion, we have described and related a number of inequivalent concepts of convergence for random dynamical systems. The weakest notion of attractor we have used is forward convergence in probability in the mean which nevertheless, by Theorem 2.8, supports all invariant measures.

Global attractors (as is the case for deterministic attractors) frequently contain large regions that are not visited asymptotically by typical trajectories and to refine the attractor we propose a notion of local attractor; the pr Milnor attractor which may be non-trivial for a much larger class of systems than the fa Milnor attractor introduced in [6].

Our investigations into random attractors of the stochastic differential equation for the multiplicatively forced van der Pol-Duffing oscillator are at the level of numerical simulations. These indicate that in particular the two-point motion can be highly intermittent and resembles intermittency near invariant subspaces studied in [8, 21].

It has been shown by Crauel and Flandoli [15] that for continuous time RDS on $\mathbf{R}$ (or order preserving discrete time systems on $\mathbf{R}$ ) the only ergodic measures are a Dirac measures supported on a random fixed point. This does not hold for RDS in higher dimensions or even continuous time RDS on the circle $\mathbf{R} / \mathbf{Z}$ [13]. Although there is a good understanding in dimension one [2] and some results in higher dimensions [4] (see [1] for a review) there remain many important unsolved problems in this area. Some of these are:

- Under what conditions can one expect to see random Milnor attractors with positive top LEs in a RDS?

- What sort of bifurcations create such random attractors, for example what happens at the boundaries of the region with positive top LEs shown in Figure 1?

- Under what circumstances (if ever) are such random Milnor attractors structurally stable?

\section{References}

[1] L. Arnold. 1998 Random Dynamical Systems. Springer Monographs in Mathematics, Springer Verlag, Berlin.

[2] L. Arnold and P. Boxler. 1990 Stochastic bifurcation: instructive examples in dimension one. in: M. Pinsky and V. Wihstutz (eds) Stochastic flows. Proceedings of Charlotte, North Carolina 1990, pp241-255. Birkhäuser, Basel.

[3] L. Arnold, Nguyen Dinh Cong and V. Oseledets. 1999 Jordan normal form for linear cocycles. Random Oper. Stoch. Equ. 7:303-358.

[4] L. Arnold and Xu Kedai. 1994 Invariant measures for random dynamical systems, a necessary condition for stochastic bifurcation from a fixed point. Random and Computational Dynamics 2:165-182. 
[5] L. Arnold, N. Sri Namachchivaya and K.R. Schenk-Hoppé. 1997 Toward an understanding of stochastic Hopf bifurcation: a case study. International Journal of Bifurcation and Chaos 6:1947-1975.

[6] P. Ashwin. 1999 Minimal attractors for random dynamical systems. Proc. Roy. Soc. London A 455:2615-2634.

[7] P. Ashwin. 1999 Attractors of a randomly forced electronic oscillator. Physica $D$ 125:302-310.

[8] P. Ashwin, J. Buescu and I.N. Stewart. 1996 From attractor to chaotic saddle: a tale of transverse instability. Nonlinearity 9:703-737.

[9] P. Baxendale. 1994 A stochastic Hopf bifurcation. Probab. Theory Relat. Fields 99:581616.

[10] P. Baxendale. 1996 Stability and equilibrium properties of stochastic flows of diffeomorphisms. To appear in: Diffusion processes and related problems in analysis, Vol II; stochastic flows. Eds M. Pinsky and V. Wihstutz. Progress in Probability, 27:3-35 Birkhäuser, Boston.

[11] H. Crauel. 1987 Random dynamical systems: Positivity of Lyapunov exponents, and Markov systems. PhD thesis, University of Bremen.

[12] H. Crauel. 2002 Random Probability Measures on Polish Spaces Stochastics Monographs Vol. 11, Taylor \& Francis, London.

[13] H. Crauel. 2002 Invariant measures for random dynamical systems on the circle. Arch. Math. 78:145-154.

[14] H. Crauel and F. Flandoli. 1994 Attractors for random dynamical systems. Prob. Theory and Related Fields 100:365-393.

[15] H. Crauel and F. Flandoli. 1998 Additive noise destroys a pitchfork bifurcation. Journal of Dynamics and Differential Eqns 10:259-274.

[16] F. Flandoli and B. Schmalfuß. 1996 Random attractors for the 3D stochastic NavierStokes equation with multiplicative white noise. Stochastics and Stochastics Reports 59:21-45.

[17] J. Guckenheimer and P. Holmes. 1983 Nonlinear oscillations, dynamical systems, and bifurcations of vector fields. Applied Mathematical Sciences 42 Springer-Verlag, New York.

[18] H. Keller and G. Ochs. 1999 Numerical approximation of random attractors. In H. Crauel and M. Gundlach (eds.): Stochastic Dynamics, Springer-Verlag, New York, pp. 93-115. 
[19] J. Milnor. 1985 On the concept of attractor. Commun. Math. Phys. 99:177-195 Comments (1985) Commun. Math. Phys. 102:517-519.

[20] G. Ochs. 1999 Weak random attractors. Report \# 449, Institut für Dynamische Systeme, Bremen.

[21] N. Platt, E.A. Spiegel and C. Tresser. 1993 On-off intermittency; a mechanism for bursting. Phys. Rev. Lett. 70:279-282.

[22] K.R. Schenk-Hoppé. 1998 Random attractors - general properties, existence, and applications to stochastic bifurcation theory. Discrete and Continuous Dynamical Systems 4:99-130.

[23] L. Schimansky-Geier and H. Herzel. 1993 Positive Lyapunov exponents in the Kramers oscillator. J. Stat. Phys. 70:141-147. 